\title{
基于CRISPR/Cas9基因编辑技术的人类遗传疾病 基因治疗相关研究进展
}

\author{
门可 ${ }^{1+*}$, 段醒妹 ${ }^{1,2 \dagger}$, 杨阳 ${ }^{1}$, 魏于全 ${ }^{1}$ \\ 1. 四川大学华西医院肿瘤中心, 四川大学生物治疗国家重点实验室, 成都 610041; \\ 2. 四川省医学科学院四川省人民医院个体化药物治疗四川省重点实验室, 成都 610072 \\ $\dagger$ 同等贡献 \\ *联系人, E-mail: mendingbob@hotmail.com
}

收稿日期: 2017-09-20; 接受日期: 2017-10-10; 网络版发表日期: 2017-11-16

国家自然科学基金(批准号: 81502677, 81602699, 81123003)、四川省科技支撑计划(批准号: 2015FZ0040)和四川省卫生和计划生育科研课 题(批准号: 16PJ488)资助

摘要 CRISPR/Cas9系统(常间回文重复序列丛集/常间回文重复序列丛集关联蛋白系统)为靶向基因编辑提 供了强大的技术手段.利用序列特异性sgRNA的引导, CRISPR/Cas9系统能够精准地在目标DNA的确切位置导 入双链切口. 与已有的基因编辑手段相比, 该系统具有更优异的简便性、特异性和有效性. 目前, 大量涉及体内 外多物种的CRISPR/Cas9基因编辑研究已充分展示了该技术的巨大潜力, 为基于该技术的疾病治疗研究和临床 应用带来了希望. 基于CRISPR/Cas9基因编辑技术所介导的非同源性末端连接和同源性DNA修复作用,近期多 个研究工作已经成功应用该技术修复了包括点突变和基因组缺失等在内的遗传疾病相关基因组缺陷. 本综述 将总结近期有关利用CRISPR/Cas9基因编辑技术治疗人类遗传性疾病的相关临床前研究进展.

关键词 CRISPR/Cas9, 基因编辑, 遗传性疾病, 基因治疗

在过去十几年中, 遗传性疾病的研究主要围绕遗 传差异这一焦点展开, 并已经取得了巨大的进展 ${ }^{[1]}$. 第 二代基因测序技术的出现和发展极大地推动了基因 组水平的相关研究, 大量与人类重大疾病相关的遗传 缺陷已被成功鉴定出来 ${ }^{[1 \sim 3]}$. 其中, 基因组序列的各种 缺陷, 如小规模序列突变、基因缺失以及大规模染色 体重排等得到了深入的探索和研究 ${ }^{[2,4,5]}$. 这些研究进 展打破了传统研究手段在遗传性疾病研究中的限制, 为基因治疗打开了新的大门 ${ }^{[1]}$.
II 型CRISPR/Cas系统编码具有核酸酶活性的标 志性Cas9蛋白, 且在三类CRISPR/Cas系统( I III)中 具有相对简单的核糖蛋白复合物构成, 因此对其机 理和应用的研究受到了高度重视 ${ }^{[6,7]}$. 在该系统中, CRISPR/Cas9核酸酶在长 $20 \mathrm{nt}$ 的sgRNA引导下准确识 别、并直接剪切目标基因 ${ }^{[8 \sim 10]}$. 与ZFN和TALEN等基 因编辑手段相比, CRISPR/Cas9的显著优点包括更加 简单、易编辑、更加高效、更低成本以及多靶点同 时进行基因编辑的潜力 ${ }^{[9,11]}$. 截至目前, 该系统已经

\footnotetext{
引用格式: 门可, 段醒妹,杨阳, 等. 基于CRISPR/Cas9基因编辑技术的人类遗传疾病基因治疗相关研究进展. 中国科学: 生命科学, 2017, 47: 1130-1140, doi: 10.1360/N052017-00169

英文版见： Men K, Duan X M, He Z Y, et al. CRISPR/Cas9-mediated correction of human genetic disease. Sci China Life Sci, 2017, 60: 447-457, doi: 10.1007/ s11427-017-9032-4
} 
被成功应用于多个物种的基因编辑研究, 在包括果蝇 (Drosophila melanogaster) $^{[12]}$ 、线虫(Caenorhabditis ele-

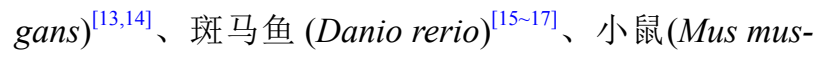
culus $)^{[8,18,19]}$ 、大鼠(Rattus norvegicus $)^{[20]}$ 以及人类 (Homo sapiens $)^{[8,11,21 \sim 23]}$ 细胞的基因组编辑研究中展现出了令 人兴奋的潜力.

CRISPR/Cas9系统在目的基因的特定位点引入双 链切割后, 细胞可通过两种主要的方式对该缺口进行 修复. 其中, 非同源性末端连接(non-homologous end joining, NHEJ) 是最为活跃的修复机制, 该修复途径具 有极大的随机性, 往往导致双链缺口区域的碱基缺 失、插入或移码突变; 另一方面, 而利用外源性修复模 板提供特定参照, 同源性DNA修复(homology directed repair, HDR)则会在目标区域按照既定目标进行准确 的修复 ${ }^{[11]}$, 从而为实现单个碱基或大片段的插入和替 换提供了可能. 更重要的是, 相较于其他现有的大部 分基因治疗策略, CRISPR/Cas9技术对基因组的改变 效应是可遗传的. 通过永久性地移除基因缺陷, 即可 实现对致病基因的彻底修复, 实现持久、稳定的治疗 效果 ${ }^{[22]}$. 因此, 近年来, CRISPR/Cas9介导的基因编辑 技术已经逐步在人类遗传性疾病的治疗研究中得到 了越来越深入的尝试和广泛的应用.

本综述将归纳近期有关CRISPR/Cas9基因编辑技 术介导的人类遗传疾病基因治疗的研究进展, 重点讨 论相关治疗策略、给药方式的设计思路, 并初步分析 相关临床治疗前景.

\section{1 修正策略}

CRISPR/Cas9基因编辑技术的多种相关功能已经 在基因治疗研究中展现出巨大的潜力. 通过在目标 DNA引入双链缺口, CRISPR/Cas9基因编辑系统可以 通过介导NHRJ或HDR作用, 产生不同类型的基因插 入、剪切或敲除, 从而实现对目标基因组序列的定向 或非定向改变. 该过程为利用CRISPR/Cas 9 技术修复 特定基因缺陷的基因治疗研究创造了机会.

\section{1 点突变修复}

外显子测序和全基因组测序极大地促进了在罕 见遗传性疾病中篎查和鉴定全新的单基因突变 $[1,24,25]$. 研究显示, 很大比例的遗传疾病是由相关致病基因特
定外显子的点突变而造成的. 而在转录过程中, 基因 的点突变将促使特殊剪切或终止信号的产生, 导致对 应mRNA或蛋白产物的减少或功能不全. 因此, 通过 CRISPR/Cas9系统在点突变位置附近引入DNA双链切 口,利用后续的同源或非同源修复作用, 可以实现对突 变引起的家族遗传疾病进行不同程度的修正和治疗.

2014年, Yin等人 ${ }^{[26]}$ 报道了利用CRISPR/Cas9技术 介导的HDR 来修复 $F a h$ 基因点突变治疗遗传性高酪氨 酸血症(type I hereditary tyrosinaemia, HTI)的研究. I 型遗传性高酪氨酸血症是一种由 $F a h$ 点突变导致的 致死性的遗传性疾病, 该基因所编码的延胡索酰乙 酰乙酸水解酶是体内酪氨酸代谢途径中最后一个参 与者 ${ }^{[26-28]}$. 该突变一般源自 HTI病人Fah 基因第 8 号外 显子上的 $\mathrm{G} \rightarrow \mathrm{A}$ 点突变, 导致基因转录时直接略过第 8 号外显子,生成欠稳定的截短性FAH蛋白产物。该缺 陷的FAH蛋白进一步引起毒性代谢产物在肝细胞蓄 积, 导致肝损伤和全身性毒性. 针对该突变, Yin等人 设计了多个靶向 Fah 基因第 8 号外显子的 $\mathrm{gRNA}$, 并将 之与 Cas9核酸酶构建于同一个质粒上共表达. 上述 CRISPR/Cas9编辑质粒与包含了野生型序列的 $199 \mathrm{nt}$ 单链脱氧核苷酸(single strand oligonucleotides, ssODN) 修复模板被一同注射到Fah5981SB小鼠(携带与人HTI 相同点突变)体内. 为了评价体内基因编辑效果, 作 者选取了包括基因测序、 $\mathrm{Fah}^{+}$细胞免疫组化分析、 mRNA定量PCR 和体重监测等一系列方法进行验证. 经过单次治疗后的小鼠的体重得到成功维持, 显示治 疗有效地保护了肝脏. 同时, 作为成功修复的直接证 据, 作者成功地从 CRISPR/Cas9治疗组小鼠体内获得 包含第 8 号外显子全长序列的PCR 条带, 并通过测序证 实 $\mathrm{A} \rightarrow \mathrm{G}$ 碱基的准确修复, 提示部分肝细胞中的第 8 9 号外显子之间恢复了正常的表达行为. 这个创新性的 研究展示了CRISPR/Cas9技术在成功修复体内基因点 突变的能力, 为CRISPR/Cas9治疗基因缺陷疾病提供 了有力的证据.

相关治疗策略也在类似的家族遗传性疾病的基 因治疗研究中进行了尝试. 例如, 通过对新生小鼠 静脉注射分别表达CRISPR/Cas9系统和DNA模板的 腺相关病毒 (adeno-associated viral vector, AAV), 杨阳 等人 ${ }^{[29]}$ 成功地在体内修复了小鼠 $O T C$ 基因第 4 号外 显子上的 $\mathrm{G} \rightarrow \mathrm{A}$ 点突变, 实现对新生小鼠高氨血症的 有效治疗. Bassuk等人 ${ }^{[30]}$ 将CRISPR/Cas9系统应用于 
编辑X连锁视网膜色素变性患者来源的诱导多能干 细胞(induced pluripotent stem cells, iPSCs), 该类患者 所携带的 $R P G R$ 基因具有 $\mathrm{c} .3070 \mathrm{G} \rightarrow \mathrm{T}$ 点突变. 该体外 基因编辑研究的修复率达到 $13 \%$. 此外, HDR介导的 点突变修复策略同样在 $\beta$ 地中海贫血 ${ }^{[9,31]}$ 、慢性肉芽 肿 ${ }^{[32]}$ 、杜氏肌营养不良(Duchenne muscular dystrophy, $\mathrm{DMD})^{[33]}$ 、镰刀型贫血 ${ }^{[34]}$ 以及重症免疫缺陷 (severe combined immunodificiency, SCID) ${ }^{[35]}$ 等其他疾病上得 到了应用。

在上述研究中, 对目的基因点突变位点的修复效 率不尽相同. 通常认为, 虽然基因序列的修复效率与 HDR 的效率呈正相关, 但是, 后者在细胞内的发生概 率总的来说较低(小于 $10 \%$ ). 因此, 设计和选择合适的 gRNA和修复模板尤为重要. gRNA在基因组上的结 合位点与CRISPR/Cas9系统的特异性和有效性密不可 分. 在 Fah 点突变修复的研究例子中, Yin等人 ${ }^{[26]}$ 设计 了 3 个靶向第 8 号外显子不同位置的 gRNA. 这些 $\mathrm{sgRNA}$ 的结合区域均直接覆盖点突变位点, 从而有利于双链 切口与之更加临近, 增加了借助 HDR 和模板进行修 复时的准确性. Long等人 ${ }^{[33]}$ 在DMD的基因编辑治疗 研究中也选取了覆盖点突变部位的gRNA. 不过, 对于 CRISPR/Cas9系统来讲, gRNA的选择受到PAM序列的 严格约束. 因此, 在一些研究案例中, 较优的靶部位可 能位于突变位点的临近区域而非直接覆盖之. 这种情 况下的CRISPR/Cas9系统被证明同样具有较高的点突 变修复效率. 例如, 杨阳等人 ${ }^{[29]}$ 在 $O T C$ 基因修复研究 中, 在位于突变位点上游和下游 $20 \sim 40 \mathrm{bp}$ 的范围内分 别设计了 3 个 $\operatorname{sgRNA}$, 并通过SURVEYOR实验篮选出 剪切效率最高的sgRNA, 而HDR介导的修复作用工作 良好. 如前所述, 即使CRISPR/Cas9系统具备产生较高 剪切效率的能力, 但细胞内HDR的发生概率通常很低. 因此, 部分CRISPR/Cas9产生的双链切口会不可避免 地通过NHEJ机制被修复, 导致体内基因编辑体有较 大概率产生不可预知的结果. 正是基于以上考虑, 杨 阳等人通过将 $\operatorname{sgRNA}$ 的靶点设计于修复位点相邻的 内含子区域, 可以有效阻止当NHEJ占上风时可能产 生的进一步编码序列的随机突变, 导致剩余OTC基因 功能的进一步缺失. 另一方面, 在治疗 SCID的基因编 辑研究中, Chang 等人 ${ }^{[35]}$ 在 $J A K 3$ 基因的第 14 号外显子 点突变位点附近设计了 6 个 $\mathrm{gRNA}$, 其中 4 个的修复效 率高达 $73.3 \%$, 而其余 2 个几乎无效. 值得一提的是, 后
2 个 sgRNA的结合区域离突变位点更远, 提示相关修 复效率的高低也可能与所选取的gRNA与突变位置距 离远近有一定关联.

除此之外, 在 HDR介导的点突变修复中, 模板 的设计策略, 如两端同源臂的长度和模板的种类也 与CRISPR/Cas 9 介导的点突变修复效率高低密切相 关. 目前已报道的相关治疗研究所运用的模板形式 多样, 其中, ssODN最为常见但长度各异. 例如, Yin 等人 ${ }^{[26]}$ 使用了长达 $199 \mathrm{nt}$ 的包含野生型序列和同源臂 的ssODN; Bassuk等人 ${ }^{[30]}$ 使用了 $162 \mathrm{nt}$ 长的ssODN作为 $\mathrm{HDR}$ 介导的 $R P G R$ 基因修复模板; Yoshimi等人 ${ }^{[2]}$ 使用 更短的 $88 \mathrm{nt} \mathrm{ssODN}$ 修复 $T y r C$ 基因的点突变. 一般来 讲, 同源臂和结合区域的长度取决于所需引入片段的 长度, 更大的修复片段可能需要更长的同源臂. 此外, dsODNs和DNA质粒也被用作修复模板参与HDR过程. 例如, Huang 等人 ${ }^{[34]}$ 使用质粒模板提供野生型 $H B B$ 基 因序列来治疗 SCID, Song等人 ${ }^{[9]}$ 构建了左臂长 $5 \mathrm{~kb}$ 、 右臂长 $3 \mathrm{~kb}$ 的质粒模板修复 $H B B$ 基因. 通常情况下, 供 体质粒在使用前需先经过限制性内切酶线性化, 再导 入细胞内发挥模板作用. 虽然使用 ssODN模板 ${ }^{[23,36,37]}$ 和 dsDNA 模板 ${ }^{[38]}$ 在HDR介导的基因编辑研究中均有 大量应用报道, 但尚无强有力的证据证明何种形式更 适合点突变修复. 同时, 由于将大片段的基因序列插 入到靶区域相较于小片段更加困难, 而各基因的修复 策略又不尽相同, 因此, HDR 修复模板的选择还需视 具体情况而定.

\section{2 基因缺失修复}

除点突变外, 遗传性疾病的另一重要致病原因为 基因组不同大小片段的碱基缺失, 其缺失范围小到单 一碱基、大到整个外显子水平. 这类基因缺陷通常会 导致可读框移码、外显子跳跃或终止信号的引入, 产 生错误或残缺的蛋白产物, 从而影响其功能. 类似于 点突变的修复, 通过利用细胞的HDR 作用, 结合适当 的修复模板, CRISPR/Cas9基因编辑技术也已经被尝 试用于该类型的遗传疾病的治疗研究.

其中一个典型的例子即是 $\mathrm{DMD}$ 的治疗. DMD是一 种由位于X染色体上的 $D m d$ 基因的突变而引起的严重 肌肉退行性疾病 ${ }^{[3,40]}$. 这些突变包括 $D m d$ 基因的小规 模碱基缺失或部分外显子的大规模丢失, 最终导致转 录过程中可读框的移码, 产生功能残缺的肌萎缩蛋白 
(dystrophin) $)^{[4]]}$. 虽然包括AAV传输 ${ }^{[2]}$ 、慢病毒传输 ${ }^{[4]}$ 以及“睡美人”转座子系统(sleeping beauty transposon) ${ }^{[43]}$ 等在内的手段已经被用于 $D M D$ 基因治疗的相关研究, 但 $D m d$ 基因较大的序列长度在很大程度上阻碍了治疗 性基因的有效递送. 因此, 力争恢复全长肌萎缩蛋白 编码基因序列的治疗努力仍然极具挑战 ${ }^{[44]}$. 同时, 一 项临床前研究结果发现, 通过调控肌萎缩蛋白mRNA 的剪切样式、实现对相关外显子区域转录的有意“忽 略”(即外显子跳跃), 可实现短暂、但部分有效的治疗 效果. 该发现也为 $D M D$ 的治疗提供了潜在的策略 ${ }^{[45]}$.

因此, 使用具有可遗传效应的基因编辑技术修复 DMD所涉及的基因缺陷被广泛认为是一种理想的治 疗手段, 并已经得到了多个研究团队的尝试. 例如, 利 用CRISPR/Cas9 系统介导的HDR功能, $\mathrm{Li}$ 等人将编码 $D m d$ 基因第 44 号外显子的全长序列敲入病人来源的 $\mathrm{iPSCs}$ 细胞中, 以替换基因组内原有的序列, 实现蛋白 质编码区的恢复. 该系统首先在第 45 号外显子中引入 双链切口, 随后由含有两个同源臂和 1 个完整的第 44 号外显子序列片段组成的载体为供体模板进行修复. 通过分析从编辑后iPSC分化得到的骨骼肌细胞, 作者 检测到了含有完整、非突变第 44 号外显子序列的肌 萎缩蛋白 mRNA, 证实所插入的第 44 号外显子与后续 的第45号外显子得以正常依次表达, 展示了该策略在 DMD治疗中的潜力.

基于CRISPR/Cas9技术的基因敲入修复方法也已 被应用于其他遗传缺陷疾病的治疗研究中. Schwank 等人使用CRISPR/Cas9技术介导的HDR 作用修复了囊 性纤维化患者来源的肠干细胞中的CFTR 基因. CFTR 基因的突变会导致其第 11 号外显子中的苯丙氨酸缺失 (CFTR F508 del), 进而导致蛋白产物错误折叠、内质 网滞留以及CFTR蛋白的过早降解 ${ }^{[46]}$. 为了修复 CFTR 基因的上述 $3 \mathrm{bp}$ 缺失, 作者将靶向第 11 号外显子或第 11 号内涵子区域的不同 $\mathrm{sgRNA}$ 与编码野生型CFTR序 列的模板质粒共同导入细胞, 并通过后续篮选和富集 得到准确修复的单克隆. 类似地, 来自两个独立课题 组的研究人员也分别报道了使用CRISPR/Cas9技术来 修复导致 $\beta$-地中海贫血的 $H B B$ 基因中的 $4 \mathrm{bp}$ 缺失 ${ }^{[31,37]}$, 而 $\mathrm{Wu}$ 等人 ${ }^{[23]}$ 则修复了导致了白内障发病的 $C r y g c$ 基因 第3号外显子中的 $1 \mathrm{bp}$ 缺失.

在上述研究中, 目标基因通过基因编辑介导的同 源性修复得以正常表达, 实现了功能完全恢复. 对于
CRISPR/Cas9系统而言, 无论需敲入的基因片段大小 如何, 一条sgRNA足以将双链切口和插入缺失(indel)引 入所需位置以诱导后续的HDR作用. 所设计的 sgRNA 的识别位点通常位于突变位点的相邻区域(当然PAM 序列的选择也需要被考虑在内). 另一方面, 前文已经 提到, 对于HDR 模板而言, 质粒DNA和ssODN各有优

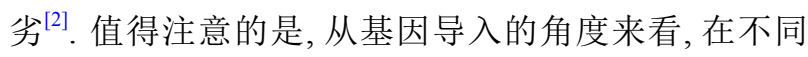
长度基因敲入的研究中, ssODN更多地被应用于涉及 受精卵或胚胎直接显微注射的相关研究手段中, 而很 少涉及利用电转的传输方法. 而多个研究报道指出, 在 使用CRISPR/Cas9系统的小鼠基因编辑研究中, ssODN 形式的供体模板在HDR介导的基因敲入时体现出了 更高的修复效率 ${ }^{[23,36-38]}$. 当用ZFN技术进行编辑时, ssODN寡核苷酸也较大型的dsDNA质粒更有效 ${ }^{[47]}$. 然 而, 据研究所知, 目前尚缺乏系统性的比较研究来分 析这两种模板形式在CRISPR/Cas9系统、特别是不同 片段长度的基因敲入应用中的优劣. 与点突变相比, 通过HDR 作用将长度为几个、几十个甚至几百个碱 基的DNA片段整合到靶位点显得更为困难和复杂 ${ }^{[2]}$, 因此需要根据实际情况来选择模板. 此外, 尽管确切 的HDR介导的修复机制仍然未知, 但 ssODN和dsDNA 各自在修复双链切口时的作用机理很可能也不尽相 同 ${ }^{[48,49]}$. 因此, 需要更多的针对 ssODN和dsDNA的系统 性研究来分析二者之间的优劣, 进而作出更加权威和 客观的判断.

\section{3 外显子切除}

CRISPR/Cas9系统还能够通过让多个gRNA 同时 发挥作用从而引入多个双链切口来实现对目的基因 进行大片段切除. 特殊情况下, 使用CRISPR/Cas9技术 进行外显子的切割也能够为相关疾病的基因治疗提 供独特的治疗思路.

DMD为这一策略在遗传性缺陷疾病治疗中的实 施提供了一个很好的例子. 如上所述, DMD 是一种导 致进行性肌营养不良的严重X连锁疾病. 目前已证实, 不同类型的基因突变参与了 $\mathrm{DMD}$ 产生, 其中, $D m d$ 基 因的一个或多个外显子的大片段缺失突变最为常见. 除了敲入缺失的 $D m d$ 基因片段的策略之外, DMD同时 还是单基因遗传性疾病中可通过剪除其基因内部不重 要的区域来实现功能修复的重要实例之一 ${ }^{[45,50]}$. 例如, $D m d$ 基因的第45 55号外显子突变常见区域中的基因 
序列缺失会导致产生截短的、但具有部分功能的肌萎 缩蛋白 ${ }^{[40]}$. 具有这种类型基因突变的患者通常是无症 状的或体现出“贝克肌营养不良症”样的轻度症状, 其 严重程度远低于 $\mathrm{DMD}^{[40]}$. 这一发现促使研究人员对于 开发基于“外显子跳跃”策略的重大兴趣. 该策略通过 调控mRNA剪切行为, 有意地“略过”特定外显子的转 录, 实现在mRNA水平将可读框恢复到正常顺序, 从而 将DMD症状转化为较轻的贝克尔样表型 ${ }^{[50]}$. 基于这一 概念, Ousterout等人尝试直接使用CRISPR/Cas9基因 编辑技术, 通过相关外显子的直接切除来治疗DMD. 他们设计了两条用于识别第 $45 \sim 55$ 号外显子外侧内含 子区域的 $\operatorname{sgRNA}$, 用于编辑DMD患者来源的骨骼成肌 细胞. 该系统成功地在相应区域产生了一截大片段的 基因组删除, 所剪下的序列长度达到 $336 \mathrm{~kb}$, 范围覆盖 第45 55号的外显子中的所有常见突变热点, 有效地解 决 $60 \%$ 以上的 $D M D$ 基因突变 ${ }^{[45,51,52]}$. 尽管该治疗策略 会导致肌萎缩蛋白的第 $45 \sim 55$ 号外显子区域缺失, 但 它有效地恢复了细胞中肌萎缩蛋白的表达, 仍然具有 积极的治疗意义. 通过富集基因编辑后的细胞并植入 免疫缺陷小鼠, 能在体内成功观察到人肌萎缩蛋白的 表达, 提示明显的治疗成果. 同样, $\mathrm{Xu}$ 等人 ${ }^{[53]}$ 也成功地 使用一对分别识别小鼠 $D m d$ 基因中第 20 和 23 号内含子 的gRNA, 实现在小鼠模型体内对第 21 号 $(181 \mathrm{bp}) 、$ 第 22 号 (146 bp)和第 23 号外显子 (213 bp)的全部切除. 此 外, 通过对DMD模型小鼠的X染色体长达 $23 \mathrm{~kb}$ 片段的 切除, 作者还成功地恢复了小鼠体内骨骼肌肌纤维膜 肌萎缩蛋白的表达.

上述研究利用对基因组中完整的外显子区域的 大规模切除, 有效实现了缺陷功能的恢复, 成功展 示了DMD治疗的新思路. 与通过NHEJ或HDR产生 indel从而修复基因缺陷的策略相比, 该方法的主要 优点是目标基因编辑后的蛋白质产物是可预测的, 并 已经在 “贝克肌营养不良症”的转化诱导研究中得到 证实. 与通过indel介导的修复相比, 该策略可有效 避免每次编辑修复过程中在目标区域产生新的、不 可预测的表型. 此外, 该方法无需使用任何形式的 供体模板予以辅助, 因此可极大地降低临床应用过 程中的成本和实施难度. 然而, 从另一个方面来看, 多个双链切口的引入不可避免地增加了对基因编辑 系统准确性的严格要求, 以降低意外染色体重排的 产生概率.

\section{4 修复染色体重排}

除点突变、基因缺失以及外显子功能缺失之外, 染色体倒置和重排也是导致遗传疾病的重要原因. CRISPR/Cas9基因编辑系统同样也已经被应用于纠正 和修复该类型的基因缺陷. 其中最具代表性的例子 是 $\mathrm{A}$ 型血友病的治疗. $\mathrm{A}$ 型血友病是由人 $F 8$ 基因突变 所引起的X连锁遗传性疾病, 由于该基因编码凝血因 子 VIII, 其突变直接导致 VIII因子关联的凝血功能障碍. 在绝大部分严重的A型血友病案例中, 将近 $1 / 2$ 是由于 $F 8$ 基因的第 1 和第 22 号内含子中的两个大型片段 (140 和600 kb)发生染色体片段重排产生 ${ }^{[54,55]}$. 该重排源自 DNA双链缺口的修复过程中偶然错误发生的非等位 基因同源重组. 针对该种基因缺陷, Park等人使用同 时含有 2 套 $\mathrm{gRNA}$ 的CRISPR/Cas9系统, 将A型血友病 患者来源的iPSC中的这两个大型重排区域同时恢复 到正常顺序. 其结果表明, 分化自编辑后iPSC细胞的 内皮细胞在体外成功完整表达 $F 8$ 基因, 同时, 血友病 小鼠模型体内的 $F 8$ 基因缺陷得到有效修复, 展示了基 于基因编辑细胞治疗的理论可行性.

在应用于基因治疗研究之前, CRISPR/Cas 9 介导 的染色体重排手段最早被用于人类疾病动物模型的建 立. 真实地在小鼠体内重现导致肿瘤发生和发展的染 色体融合或重排情况, 将为临床用药的有效性和敏感 性提供强有力的支撑 ${ }^{[56-58]}$. 最近, 由Maddalo和Blasco分 别领导的两个独立的研究小组 ${ }^{[7,59]}$ 通过CRISPR/Cas9 基因编辑手段成功地在小鼠体内建立了由Eml4-Alk 基因重排而驱动的肺癌模型. Choi和Meyerson ${ }^{[60]}$ 也 成功地利用CRISPR/Cas9技术在小鼠体内重现了包括 CD74-ROS1, EML4-ALK和KIF5B-RET在内的多个驱 动肺癌发生发展的染色体重排现象. 这些研究为建立 准确的疾病基因模型以及基因缺陷研究方法提供了 更加灵活和简便的策略. 此外, 上述相关A型血友病的 治疗研究成果也开创性地证明, CRISPR/Cas9基因编 辑技术可以有效修复患者来源的 $\mathrm{PSC}$ 细胞中的染色 体倒置或大片段重排. 该成果为同样涉及染色体倒置 的亨特综合征 ${ }^{[6]}$ 和癌症 ${ }^{[62]}$ 等其他遗传疾病的治疗提 供了潜在的手段和新的希望.

\section{CRISPR/Cas9系统导入策略}

除治疗策略之外, 成功而高效的CRISPR/Cas9系 
统导入途径也对相关疾病的治疗效果起着决定性的 作用. 截至目前, 已有多个研究成功展示了运用不同 传输手段对各种形式的CRISPR/Cas9基因编辑系统予 以传输的实例, 均取得了不同程度的治疗效果. 在这 些研究中, iPSC细胞的编辑和诱导、体内系统性传输 以及受精卵的显微注射 3 种策略的应用得到了较多的 关注.

\section{1 基于iPSC 细胞的基因编辑}

iPSC技术的出现和迅速发展为个体化细胞治疗 带来了新的希望 ${ }^{[63-67]}$. iPSC作为一种独特的、可分化 的多功能细胞来源, 可通过其潜在的强大分化能力修 复损伤或患病组织, 进而实现对广泛疾病的治疗 ${ }^{[68,69]}$. 基因编辑手段通过对iPSC细胞的基因改造, 能够实现 对iPSC细胞的分化前修饰, 进而获得更为优化的细胞 功能. 因此, iPSC和CRISPR/Cas9技术的结合也为遗传 疾病的治疗带来了新的思路和希望 ${ }^{[70]}$. 编辑和制备具 有患者个体特异性的iPSC细胞作为个体化再生医学 的长期目标, 将为生理功能研究、药物篮选、治疗效 果评估以及基于基因修复的细胞替代疗法提供特有 的平台 ${ }^{[71,72]}$.

近期研究表明, 经过基因编辑的患者特异性iPSC 为 $\beta$-地中海贫血、SCID、视网膜色素变性和 $\mathrm{A}$ 型血 友病等遗传性疾病的治疗提供了创新的策略. 例如, Xie 等人 ${ }^{[31]}$ 利用 CRISPR/Cas9技术修复了 $\beta$-地中海贫 血患者来源的iPSC细胞中 $H B B$ 基因的突变. 通过篮 选编辑后的iPSC 并进一步使其分化为成红血细胞, 所获得的细胞群表现出对 $H B B$ 基因的正常表达. 同 时, 取自患者自身体细胞的iPSC细胞为基因编辑和 修复提供了丰富的细胞来源, 而所获得的iPSC可以进 一步分化为可用于自体移植的造血干细胞和祖细胞 (hematopoieticstem/progenitorcell, HSPC), 为后续体内 研究提供了良好基础. 这种方法将有效地避免诸如同 种异体移植所带来的免疫排斥反应或由病毒载体引 入的基因整合等安全性问题 ${ }^{[31]}$.

类似的, $\mathrm{Li}$ 等人 ${ }^{[44]}$ 在源自DMD患者的iPSC中进行 了3 种不同的基因编辑尝试, 最终成功获得分化为可 表达全长肌萎缩蛋白的骨骼肌细胞. 利用SCID患者来 源的特异性iPSC细胞, Chang等人 ${ }^{[35]}$ 通过CRISPR/Cas9 技术修复了 $J A K 3$ 基因突变, 并成功恢复了 $\mathrm{T}$ 细胞的正 常发育功能. 类似的基因治疗策略也被成功应用于从
$\mathrm{A}$ 型血友病 ${ }^{[55}$ 和视网膜色素变性 ${ }^{[30]}$ 患者体内分离得到 的iPSC中. 上述基于CRISPR/Cas9技术的研究为利用 患者来源的iPSC 细胞开展更为先进的细胞和基因治 疗提供了理论基础. 该疗法不仅可省去免疫抑制药物 的使用，同时通过与CRISPR/Cas9技术介导的多种基 因修复策略相结合, 还能实现对缺陷基因和功能的精 准修复, 获得“叠加”的治疗效果 ${ }^{[71]}$.

\section{2 体内系统性传输}

为了实现基于CRISPR/Cas9技术的体内基因编辑, 包括静脉注射 ${ }^{[73]}$ 、肺内注射 ${ }^{[59]}$ 、眼球后静脉注射 ${ }^{[74]}$ 和前额叶皮质注射 ${ }^{[75]}$ 等在内的技术手段已被用于基 因编辑组分的体内传递. 其中, 借助相关策略在小鼠 体内引入基因敲除、重排以及敲入等的研究手段最 为常见. 这些研究成果进一步促进了体内系统性传 输CRISPR/Cas9系统治疗人类遗传性疾病的相关研究 尝试.

如前文所述, I 型遗传性高酪氨酸血症是一种由 FAH蛋白缺乏引起的遗传疾病. 该疾病导致肝细胞中 有毒代谢物的积累, 进而产生严重的肝损伤 ${ }^{[28]}$. 前期研 究表明, 通过 $\mathrm{AAV}$ 载体静脉输送野生型F $h$ 基因序列 可在小鼠体内实现稳定的基因修复, 但是, 其传输载体 AAV所涉及的基因组整合特性是一个不可回避的安全 问题 ${ }^{[28]}$. 另一方面, 有研究报道称肝脏中已获得 $F a h$ 基 因修复的肝细胞相对于非编辑细胞具有选择性增殖优 势, 其细胞群可通过逐步分裂扩增实现最终重建肝脏 功能 ${ }^{[28]}$. 受此提示, Yin等人 ${ }^{[26]}$ 通过尾静脉高压注射法 将Fah 基因靶向的CRISPR/Cas9系统质粒和对应 $\mathrm{SSODN}$ 修复模板导入酪氨酸血症小鼠中 $\left(\mathrm{Fah}^{\text {mut } m u t}\right)$, 以期在肝 脏中进行基因修复治疗. 通过对治疗后小鼠的肝脏样 本和包括天冬氨酸转氨酶和丙氨酸氨基转移酶在内的 血清标志物的分析, 作者观察到了CRISPR/Cas9系统对 肝脏中 Fah 突变的成功修复, 实现对因FAH蛋白缺乏而 引起的肝损伤的有效治疗. 在该研究中, CRISPR/Cas9 系统的体内递送通过尾静脉高压注射法实现. 该方法 通过尾静脉快速注射大体积的质粒DNA溶液, 从而简 单而高效地实现目的基因的肝组织分布 ${ }^{[76]}$. 近年来, 通 过尾静脉高压注射法传输治疗基因到肝组织的相关 机制已经得到了深入的研究 ${ }^{[77]}$, 并已经被应用于包括 $\mathrm{HBV}^{[78]}$ 和血友病 ${ }^{[79]}$ 等在内的多种基于质粒DNA的基 因治疗研究中. 在Yin等人的工作中, 尾静脉高压注射 
的方法促进了CRISPR/Cas9质粒复合物在小鼠体内对 肝组织的有效递送, 实现 $0.40 \% \pm 0.12 \%$ 的体内基因修 复率, 体现出一定的应用潜力. 然而, 由于对人体血液 循环施加过重的负载容易引起心脏功能的损伤, 并导 致短暂的心衰 ${ }^{[77]}$, 因此借助全身血液循环系统的尾静 脉高压注射手段在人体内实施的可行性较低. 虽然啮 齿类动物对该副作用耐受性较好, 但对于患者来讲可 能并不安全. 因此, 从临床应用的观点来看, 尚有待于 进一步开发更安全的CRISPR/Cas9系统体内导入手段.

在另一项体内治疗研究案例中, 为了纠正类似的 基因突变以实现治疗目的, 杨阳等人 ${ }^{[29]}$ 将OTC 基因靶 向的CRISPR/Cas9系统通过静脉注射导入到OTC缺陷 的新生小鼠中. 在该研究中, 他们将源自金黄色葡萄 球菌的CRISPR/Cas9系统(SaCas9)整合到两个AAV8载 体中, 一个载体用于 $\mathrm{SaCas} 9$ 的表达, 另一个提供相应 gRNA和修复模板. 为了能够更好地评估在小鼠生长 过程中 $O T C$ 基因的修复所产生的治疗作用, 其作者巧 妙地使用新生小鼠进行试验. 其结果表明, 肝脏OTC酶 活性随着编辑后细胞群的增殖扩增, 在第3 8周的观察 区间内获得了明显的恢复. 在上述研究中, 作者使用了 8 型腺相关病毒载体(AAV8)作为基因编辑系统的体内 肝脏递送系统. 不同血清型的腺相关病毒能够作为针 对不同人体组织的基因递送载体而提供长期而强大的 基因导入能力 ${ }^{[80-82]}$. 目前已有部分基于 $A A V$ 的基因治 疗产品被批准进入临床使用 ${ }^{[83,84]}$. 而经门静脉注射后, AAV8病毒载体被报道可感染小鼠肝脏中 $90 \%$ \% $95 \%$ 的 细胞 ${ }^{[84]}$. 通过使用高度肝脏亲和力的AAV8载体, 杨阳 及其同事在利用CRISPR/Cas9技术实现肝脏代谢疾病 的基因治疗研究中实现了突破. 其较高的体内修复效 率同时还得益于在细胞不断分裂的背景下, $\mathrm{AAV}$ 载体 对 $\mathrm{SaCas} 9$ 系统和修复模板高效而持续的整合表达.

除了上述研究外, AAV载体也已被成功地应用在 多个CRISPR/Cas9介导的体内基因编辑研究中, 展现 出令人印象深刻的效果和潜力 ${ }^{[73,85,86]}$. 不过, 关于该载 体的进一步应用仍然存在一些值得关注的问题. 例如, 多个临床前和临床研究显示, AAV8载体对小鼠肝脏 的基因传输效率比人体高 20 倍, 推测在人体内的应用 可能达不到动物模型相同理想的效果 ${ }^{[87 \sim 90]}$. 使用 AAV 载体的另一担忧是其宿主基因组整合能力, 而该能力 并非是实施CRISPR/Cas9基因编辑所必需的, 且有可 能导致免疫系统毒性或基因组损伤. 不过即便如此,
上述研究依然成功地为体内静脉传输CRISPR/Cas9系 统介导治疗人类致死性代谢疾病提供了令人信服的 理论和实践依据.

\section{3 受精卵显微注射}

受精卵显微注射技术已经被广泛应用于各种动物 模型的建模中. 目前, 大多数转基因动物均是通过将 目的基因引入受精卵实现, 相关的技术和支撑学科也 得到了长足的发展 ${ }^{[91]}$. 通过将CRISPR/Cas9系统注射 到受精卵或早期胚胎中, 可以实现对生物个体包括生 殖细胞在内的所有细胞基因组的编辑 ${ }^{[2]}$. 理论上, 这种 方法可实现对所有体细胞和生殖细胞的永久性改变, 从而成功将编辑后的基因型稳定遗传给后代. 因此, 受 精卵注射的传输手段已经被应用于基于CRISPR/Cas9 技术的动物建模和体内基因治疗中.

例如, 为了修复 $C r y g c$ 基因的突变, $\mathrm{Wu}$ 等人 ${ }^{[23]}$ 将 CRISPR/Cas9系统以 mRNA和gRNA复合物的形式, 通 过显微注射导入小鼠的受精卵细胞中. 其所编辑的 受精卵取自携带白内障致病基因的 $\mathrm{Crygc}^{-/}$小鼠与野 生型小鼠的杂交后代. 在22只新生幼仔中, 作者鉴定 得到了 10 只携带编辑后 $C r y g c$ 基因的小鼠, 而其中 4 只 小鼠成功通过HDR诱导的等位基因修复, 避免了白内 障表型的产生. 在DMD的治疗研究中, Long等人 ${ }^{[33]}$ 将 组成CRISPR/Cas9系统的mRNA, gRNA和ssODN同源 修复模板共同注射到小鼠受精卵中, 以纠正 $m d x$ 缺陷 小鼠染色体中的 $D m d$ 基因突变. 其所获得的 11 个后代 中, 有7只小鼠被检测到在 $D m d$ 的第 23 号外显子上发 生了HDR介导的基因修复, 另外4只小鼠含有由NHEJ 介导的可读框内终止密码子移除. 其研究结果表明, CRISPR/Cas9技术介导的受精卵基因编辑可产生具有 不同基因型的后代, 其目标基因 $D m d$ 的修复率范围为 $2 \% \sim 100 \%$. 更为有趣的是, 通过进一步深入研究发现, 所获得的基因修复效果还具有时间依赖的特征, 并且 该现象只有在骨骼肌中才能观察到. 以上研究表明, 利用CRISPR/Cas9系统对受精卵进行基因编辑在相关 疾病的基因治疗研究中体现出了较高的安全性和有 效性.

一些表现为系统性呈现的遗传性基因疾病(如囊 性纤维化或遗传性线粒体疾病)、单基因病变但影响 广泛的遗传性基因疾病(如肌营养不良)或治疗手段难 以触及的遗传性基因疾病(如亨廷顿氏病中涉及的基 
底神经节)的治疗对于基于体细胞的治疗方法来说可 能往往具有较大挑战性 ${ }^{[33]}$. 因此, 针对受精卵或胚胎 的基因组编辑很可能会为上述疾病的治疗提供更好 的选择. 然而, 即使将伦理学考量放在一边, 多个关键 的问题仍然限制着在人体上实施基于受精卵的基因 编辑. 例如, 有研究显示, 在通过显微注射、并已经实 现了基因组编辑的细胞在进一步有丝分裂时, 所得到 的子代细胞的基因组之间仍然可能出现基因型的异 质性 ${ }^{[33,92]}$, 这使得对基因编辑所产生的后代的表型进 行合理预测和控制变得困难. 同时, 虽然CRISPR/Cas9 系统可通过显微注射成功导入并实施编辑, 但其编 辑效率并不完全可控, 导致所产生的后代可能为嵌 合子, 即有一部分体细胞和生殖细胞可能并不具有 编辑后的基因组成 ${ }^{[92]}$. 此外, 还有部分学者报道称将 CRISPR/Cas9系统直接注射到受精卵中可能降低生物 个体产生健康后代的几率 ${ }^{[23]}$. 更重要的是, 目前, 在人 体上进行生殖细胞或受精卵内的基因组编辑是被广 泛禁止的 ${ }^{[33,92]}$. 因此, 必须制定更为明确的伦理准则, 进一步通过多种角度讨论基因组编辑对社会影响 ${ }^{[33]}$. 然而, 如果单从技术层面上看, 现有的基于小鼠受精 卵的基因编辑研究仍然表明CRISPR/Cas9 介导基因治 疗巨大潜力.

\section{3 展望}

CRISPR/Cas9技术为编辑特定基因提供了强大的 工具, 目前已经在遗传疾病的治疗中展现出了巨大的 应用潜力. 不论是通过体内系统性给药(如使用疾病 动物模型)或离体递送(如体外iPSC 细胞编辑), 使用 CRISPR/Cas9技术来纠正基因缺陷的体内外相关研究 已取得显着的进步, 为临床应用基因编辑的手段进行 遗传病基因治疗带来了更多希望. 同时, 后续的相关研 究将会在CRISPR/Cas9基因编辑技术用于基因治疗的 有效性、特异性和安全性问题上开展更为深入的研究, 进一步挑战相关技术难点. 这些挑战包括但不限于: ( i ) 开发更优的基因传递系统用于CRISPR/Cas9系统 的体内递送; (ii) 进一步发掘AAV和新型非病毒基因 载体的应用潜力; (iii) 在CRISPR/Cas9系统的设计过 程中提高HDR介导的基因修复的效率, 减少NHEJ导致 的非目标突变; (iv) 以及进一步评估不同治疗和给药 途径对于基因编辑系统的组织特异性的影响. 此外, 基 于基因编辑的临床前研究还应特别关注CRISPR/Cas9 系统的脱靶问题. 尽管存在这些挑战, CRISPR/Cas9基 因编辑技术的快速发展将最终促进人类遗传疾病基 因治疗的不断进步.

\section{参考文献}

1 Veltman J A, Brunner H G. De novo mutations in human genetic disease. Nat Rev Genet, 2012, 13: $565-575$

2 Yoshimi K, Kaneko T, Voigt B, et al. Allele-specific genome editing and correction of disease-associated phenotypes in rats using the CRISPR-Cas platform. Nat Commun, 2014, 5: 4240

3 Ott J, Kamatani Y, Lathrop M. Family-based designs for genome-wide association studies. Nat Rev Genet, 2011, 12: 465-474

4 Beckmann J S, Estivill X, Antonarakis S E. Copy number variants and genetic traits: closer to the resolution of phenotypic to genotypic variability. Nat Rev Genet, 2007, 8: 639-646

5 Frazer K A, Murray S S, Schork N J, et al. Human genetic variation and its contribution to complex traits. Nat Rev Genet, 2009, 10: 241-251

6 Zhang X, Wang S. From the first human gene-editing to the birth of three-parent baby. Sci China Life Sci, 2016, 59: 1341-1342

7 Zhang D, Li Z, Yan B, et al. A novel RNA-guided RNA-targeting CRISPR tool. Sci China Life Sci, 2016, 59: 854-856

8 Cong L, Ran F A, Cox D, et al. Multiplex genome engineering using CRISPR/Cas systems. Science, 2013, 339: 819-823

9 Song B, Fan Y, He W, et al. Improved hematopoietic differentiation efficiency of gene-corrected beta-thalassemia induced pluripotent stem cells by CRISPR/Cas9 System. Stem Cells Dev, 2015, 24: 1053-1065

10 Zhang D, Li J F. DNA-guided genome editing tool. Sci China Life Sci, 2016, 59: 740-741

11 Mali P, Yang L, Esvelt K M, et al. RNA-guided human genome engineering via Cas9. Science, 2013, 339: 823-826

12 Yu Z, Ren M, Wang Z, et al. Highly efficient genome modifications mediated by CRISPR/Cas9 in Drosophila. Genetics, 2013, 195: 289-291

13 Dickinson D J, Ward J D, Reiner D J, et al. Engineering the Caenorhabditis elegans genome using Cas9-triggered homologous recombination. Nat Methods, 2013, 10: 1028-1034

14 Friedland A E, Tzur Y B, Esvelt K M, et al. Heritable genome editing in C. elegans via a CRISPR-Cas9 system. Nat Methods, 2013, 10: 741-743 
19 Gao X. Model animals and their applications. Sci China Life Sci, 2015, 58: 319-320

\section{Biotechnol, 2013, 31: 684-686} 2013, 110: 13904-13909 31: $230-232$ 551-553 903-910 2010, 51: 1200-1208 Biotechnol, 2016, 34: 334-338 Genome Res, 2014, 24: 1526-1533 human iPS cells. Exp Hematol, 2015, 43: 838-848.e3 Science, 2014, 345: 1184-1188 after genome editing of the sickle point mutation. Stem Cells, 2015, 33: 1470-1479 targeting. Cell Rep, 2015, 12: 1668-1677 engineering. Cell, 2013, 153: 910-918 engineering. Cell, 2013, 154: 1370-1379 Duchenne muscular dystrophy. Nat Commun, 2015, 6: 6244 2011, 19: 830-840 Pharmaceuticals, 2013, 6: 813-836 Commun, 2013, 4: 1549 stem cells by TALEN and CRISPR-Cas9. Stem Cell Rep, 2015, 4: 143-154

Chang N, Sun C, Gao L, et al. Genome editing with RNA-guided Cas9 nuclease in zebrafish embryos. Cell Res, 2013, 23: 465-472

Hwang W Y, Fu Y, Reyon D, et al. Efficient genome editing in zebrafish using a CRISPR-Cas system. Nat Biotechnol, 2013, 31: 227-229

Cho S W, Kim S, Kim J M, et al. Targeted genome engineering in human cells with the Cas9 RNA-guided endonuclease. Nat Biotechnol, 2013,

Jinek M, East A, Cheng A, et al. RNA-programmed genome editing in human cells. Elife, 2013, 2: e00471

Savic N, Schwank G. Advances in therapeutic CRISPR/Cas9 genome editing. Trans Res, 2016, 168: 15-21

Wu Y, Liang D, Wang Y, et al. Correction of a genetic disease in mouse via use of CRISPR-Cas9. Cell Stem Cell, 2013, 13: 659-662

Bamshad M J, Ng S B, Bigham A W, et al. Exome sequencing as a tool for Mendelian disease gene discovery. Nat Rev Genet, 2011, 12: 745-755

Gilissen C, Hoischen A, Brunner H G, et al. Unlocking Mendelian disease using exome sequencing. Genome Biol, 2011, 12: 1

Yin H, Xue W, Chen S, et al. Genome editing with Cas9 in adult mice corrects a disease mutation and phenotype. Nat Biotechnol, 2014, 32:

Azuma H, Paulk N, Ranade A, et al. Robust expansion of human hepatocytes in Fah-/-/Rag2-/-/I12rg-/- mice. Nat Biotechnol, 2007, 25:

Paulk N K, Wursthorn K, Wang Z, et al. Adeno-associated virus gene repair corrects a mouse model of hereditary tyrosinemia in vivo. Hepatology,

Yang Y, Wang L, Bell P, et al. A dual AAV system enables the Cas9-mediated correction of a metabolic liver disease in newborn mice. Nat

Bassuk A G, Zheng A, Li Y, et al. Precision medicine: genetic repair of retinitis pigmentosa in patient-derived stem cells. Sci Rep, 2016, 6: 19969

Xie F, Ye L, Chang J C, et al. Seamless gene correction of $\beta$-thalassemia mutations in patient-specific iPSCs using CRISPR/Cas9 and piggyBac.

Flynn R, Grundmann A, Renz P, et al. CRISPR-mediated genotypic and phenotypic correction of a chronic granulomatous disease mutation in

3 Long C, McAnally J R, Shelton J M, et al. Prevention of muscular dystrophy in mice by CRISPR/Cas9-mediated editing of germline DNA.

4 Huang X, Wang Y, Yan W, et al. Production of gene-corrected adult beta globin protein in human erythrocytes differentiated from patient iPSCs

Chang C W, Lai Y S, Westin E, et al. Modeling human severe combined immunodeficiency and correction by CRISPR/Cas9-enhanced gene

Wang H, Yang H, Shivalila C S, et al. One-step generation of mice carrying mutations in multiple genes by CRISPR/Cas-mediated genome

Yang H, Wang H, Shivalila C S, et al. One-step generation of mice carrying reporter and conditional alleles by CRISPR/Cas-mediated genome

Carbery I D, Ji D, Harrington A, et al. Targeted genome modification in mice using zinc-finger nucleases. Genetics, 2010, 186: 451-459

Hoffman E P, Brown R H, Kunkel L M. Dystrophin: the protein product of the Duchenne muscular dystrophy locus. Cell, 1987, 51: 919-928

Ousterout D G, Kabadi A M, Thakore P I, et al. Multiplex CRISPR/Cas9-based genome editing for correction of dystrophin mutations that cause

1 Pichavant C, Aartsma-Rus A, Clemens P R, et al. Current status of pharmaceutical and genetic therapeutic approaches to treat DMD. Mol Ther,

Okada T, Takeda S I. Current challenges and future directions in recombinant AAV-mediated gene therapy of Duchenne muscular dystrophy.

3 Filareto A, Parker S, Darabi R, et al. An ex vivo gene therapy approach to treat muscular dystrophy using inducible pluripotent stem cells. Nat

Li H L, Fujimoto N, Sasakawa N, et al. Precise correction of the dystrophin gene in duchenne muscular dystrophy patient induced pluripotent 
Aartsma-Rus A, Fokkema I, Verschuuren J, et al. Theoretic applicability of antisense-mediated exon skipping for Duchenne muscular dystrophy mutations. Hum Mutat, 2009, 30: 293-299

46 Cheng S H, Gregory R J, Marshall J, et al. Defective intracellular transport and processing of CFTR is the molecular basis of most cystic fibrosis. Cell, 1990, 63: 827-834

47 Chen F, Pruett-Miller S M, Huang Y, et al. High-frequency genome editing using ssDNA oligonucleotides with zinc-finger nucleases. Nat Meth, 2011, 8: 753-755

48 Weiner A, Zauberman N, Minsky A. Recombinational DNA repair in a cellular context: a search for the homology search. Nat Rev Microbiol, 2009, 7: 748-755

49 Huertas P. DNA resection in eukaryotes: deciding how to fix the break. Nat Struct Mol Biol, 2010, 17: 11-16

50 Lu Q L, Yokota T, Takeda S I, et al. The status of exon skipping as a therapeutic approach to duchenne muscular dystrophy. Mol Ther, 2011, 19: 9-15

51 Aartsma-Rus A, Kaman W E, Weij R, et al. Exploring the frontiers of therapeutic exon skipping for Duchenne muscular dystrophy by double targeting within one or multiple exons. Mol Ther, 2006, 14: 401-407

52 Aoki Y, Yokota T, Nagata T, et al. Bodywide skipping of exons 45-55 in dystrophic mdx52 mice by systemic antisense delivery. Proc Natl Acad Sci USA, 2012, 109: 13763-13768

$53 \mathrm{Xu} \mathrm{L}$, Park K H, Zhao L, et al. CRISPR-mediated genome editing restores dystrophin expression and function in $m d x$ mice. Mol Ther, 2016, 24: 564-569

54 Graw J, Brackmann H H, Oldenburg J, et al. Haemophilia A: from mutation analysis to new therapies. Nat Rev Genet, 2005, 6: 488-501

55 Park C Y, Kim D H, Son J S, et al. Functional correction of large factor VIII gene chromosomal inversions in hemophilia A patient-derived iPSCs using CRISPR-Cas9. Cell Stem Cell, 2015, 17: 213-220

56 Sharpless N E, DePinho R A. The mighty mouse: genetically engineered mouse models in cancer drug development. Nat Rev Drug Discov, 2006, 5: $741-754$

57 Maddalo D, Manchado E, Concepcion C P, et al. In vivo engineering of oncogenic chromosomal rearrangements with the CRISPR/Cas9 system. Nature, 2014, 516: 423-427

58 Pirazzoli V, Nebhan C, Song X, et al. Acquired resistance of EGFR-mutant lung adenocarcinomas to afatinib plus cetuximab is associated with activation of mTORC1. Cell Rep, 2014, 7: 999-1008

59 Blasco R B, Karaca E, Ambrogio C, et al. Simple and rapid in vivo generation of chromosomal rearrangements using CRISPR/Cas9 technology. Cell Rep, 2014, 9: 1219-1227

60 Choi P S, Meyerson M. Targeted genomic rearrangements using CRISPR/Cas technology. Nat Commun, 2014, 5: 3728

61 Bondeson M L, Dahl N, Malmgren H, et al. Inversion of the IDS gene resulting from recombination with IDS-related sequences in a common cause of the Hunter syndrome. Hum Mol Genet, 1995, 4: 615-621

62 Nikiforova M N, Stringer J R, Blough R, et al. Proximity of chromosomal loci that participate in radiation-induced rearrangements in human cells. Science, 2000, 290: 138-141

63 Takahashi K, Yamanaka S. Induction of pluripotent stem cells from mouse embryonic and adult fibroblast cultures by defined factors. Cell, 2006, 126: $663-676$

64 Hanna J, Wernig M, Markoulaki S, et al. Treatment of sickle cell anemia mouse model with iPS cells generated from autologous skin. Science, 2007, 318: 1920-1923

Park I H, Arora N, Huo H, et al. Disease-specific induced pluripotent stem cells. Cell, 2008, 134: 877-886

Raya A, Rodríguez-Pizà I, Guenechea G, et al. Disease-corrected haematopoietic progenitors from Fanconi anaemia induced pluripotent stem cells. Nature, 2009, 460: 53-59

67 Mukherjee S, Thrasher A J. iPSCs: unstable origins? Mol Ther, 2011, 19: 1188

68 Avior Y, Sagi I, Benvenisty N. Pluripotent stem cells in disease modelling and drug discovery. Nat Rev Mol Cell Biol, 2016, 17: 170-182

Chen Z G, Zhang Y A. Cell therapy for macular degeneration-first phase I/II pluripotent stem cell-based clinical trial shows promise. Sci China Life Sci, 2015, 58: 119-120

70 Kimbrel E A, Lanza R. Current status of pluripotent stem cells: moving the first therapies to the clinic. Nat Rev Drug Discov, 2015, 14: 681-692

71 Robinton D A, Daley G Q. The promise of induced pluripotent stem cells in research and therapy. Nature, 2012, 481: 295-305

72 Trounson A, DeWitt N D. Pluripotent stem cells progressing to the clinic. Nat Rev Mol Cell Biol, 2016, 17: 194-200

73 Ran F A, Cong L, Yan W X, et al. In vivo genome editing using Staphylococcus aureus Cas9. Nature, 2015, 520: 186-191

74 Ding Q, Strong A, Patel K M, et al. Permanent alteration of PCSK9 with in vivo CRISPR-Cas9 genome editing. Circul Res, 2014, 115: 488-492 


\section{5, 23: 1002-1007}

79 Olivares E C, Hollis R P, Chalberg T W, et al. Site-specific genomic integration produces therapeutic Factor IX levels in mice. Nat Biotechnol, 2002, 20: 1124-1128

80 Inagaki K, Fuess S, Storm T A, et al. Robust systemic transduction with AAV9 vectors in mice: efficient global cardiac gene transfer superior to that of AAV8. Mol Ther, 2006, 14: 45-53

81 Zincarelli C, Soltys S, Rengo G, et al. Analysis of AAV serotypes 1-9 mediated gene expression and tropism in mice after systemic injection. Mol Ther, 2008, 16: 1073-1080

82 Asokan A, Schaffer D V, Samulski R J. The AAV vector toolkit: poised at the clinical crossroads. Mol Ther, 2012, 20: 699-708

83 Mingozzi F, High K A. Therapeutic in vivo gene transfer for genetic disease using AAV: progress and challenges. Nat Rev Genet, 2011, 12: $341-355$

84 Piras B A, Drury J E, Morton C L, et al. Distribution of AAV8 particles in cell lysates and culture media changes with time and is dependent on the recombinant vector. Mol Ther Methods Clin Dev, 2016, 3: 16015

85 Swiech L, Heidenreich M, Banerjee A, et al. In vivo interrogation of gene function in the mammalian brain using CRISPR-Cas9. Nat Biotechnol, 2015, 33: 102-106

86 Shinmyo Y, Tanaka S, Tsunoda S, et al. CRISPR/Cas9-mediated gene knockout in the mouse brain using in utero electroporation. Sci Rep, 2017, in press, doi: 10.1002/cpns.26

87 Davidoff A M, Gray J T, Ng C Y C, et al. Comparison of the ability of adeno-associated viral vectors pseudotyped with serotype 2, 5, and 8 capsid proteins to mediate efficient transduction of the liver in murine and nonhuman primate models. Mol Ther, 2005, 11: 875-888

88 Kay M A, Manno C S, Ragni M V, et al. Evidence for gene transfer and expression of factor IX in haemophilia B patients treated with an AAV vector. Nat Genet, 2000, 24: 257-261

89 Lisowski L, Dane A P, Chu K, et al. Selection and evaluation of clinically relevant AAV variants in a xenograft liver model. Nature, 2014, 506: 382

90 Manno C S, Pierce G F, Arruda V R, et al. Successful transduction of liver in hemophilia by AAV-Factor IX and limitations imposed by the host immune response. Nat Med, 2006, 12: 342-347

91 Smith K R. Gene therapy: the potential applicability of gene transfer technology to the human germline. Int J Med Sci, 2004, 1: 76-91

92 Porteus M H, Dann C T. Genome editing of the germline: broadening the discussion. Mol Ther, 2015, 23: 980-982 